\title{
APPLICATION OF KHAS'MINSKII'S LIMIT THEOREM TO THE BUCKLING PROBLEM OF A COLUMN WITH RANDOM INITIAL DEFLECTIONS*
}

\author{
By B. P. VIDEC AND J. LYELL SANDERS, JR. (Harvard University)
}

\begin{abstract}
An approximate asymptotic expression is obtained for the buckling load of an imperfect column resting on a nonlinear elastic foundation. The result holds for a large range of imperfection shapes, which are assumed to be stationary random functions of position. The asymptotic analysis is based on application of Khas'minskii's limit theorem to equations for the slowly varying part of the deflection of the column. Previous results obtained for Gaussian imperfection shapes are shown to be valid also for the larger class of random imperfections considered here.
\end{abstract}

Introduction. In this paper the buckling behavior of a uniform column resting on a softening nonlinear foundation will be studied. The imperfect column to be considered will have small stress-free initial deflections which are stationary random functions of position.

In earlier studies of this imperfection sensitive model structure Fraser and Budiansky [1], and Amazigo, Budiansky and Carrier [2] obtained asymptotic expressions for the buckling load by means of both equivalent linearization and truncated-hierarchy methods. In a subsequent study [3] Amazigo used a perturbation scheme to arrive at a slightly different result for the buckling load. In each of these studies the initial deflection was assumed to be a stationary Gaussian random function of position.

The asymptotic analysis presented here is based upon a limit theorem due to Khas'minskii [5]. The shape of the imperfection is assumed to belong to the class of random functions considered in [5]. The asymptotic results found in [3] for the buckling load of columns with Gaussian imperfection shapes are shown to be valid also for the larger class of random functions considered here.

Differential equation. The differential equation governing the nondimensionalized lateral displacement $w(x)$ of a column of length $2 l$ on a softening nonlinear elastic foundation is $[2,3]$.

$$
w^{\prime \prime \prime \prime}+2 \lambda w^{\prime \prime}+w-w^{3}=-2 \lambda \epsilon w_{0}^{\prime \prime} \quad-l<x<l
$$

where ()$^{\prime}=d / d x()$. The column, which is subjected to the dimensionless axial load $\lambda$, is restrained against lateral displacement by a restoring force per unit length $w-w^{3}$. The stress-free initial displacement $w_{0}(x)$ is a bounded, zero mean, stationary random function of position and $\epsilon$ is a small imperfection parameter. The column is taken to be hinged (simply supported) at both ends with boundary conditions: $w=w^{\prime \prime}=0$ at

* Received October 6, 1974. This work was supported in part by the Air Force Office of Scientific Research under Grant AFOSR-73-2476, in part by the National Science Foundation under Grant GP-34723, and by the Division of Engineering and Applied Physics, Harvard University. 
$x= \pm l$. Note that in the differential equation the additional displacement $w(x)$ is assumed to be small enough that the nonlinear terms resulting from the kinematic conditions can be neglected.

The solution of the nonlinear, nonhomogeneous equation (1) would provide a relation between the load parameter $\lambda$, the displacement $w$ and the imperfection parameter $\epsilon$. For the branch of solutions satisfying the condition $w=0$ for $\lambda=0$, the load $\lambda$ is expected to reach a maximum value $\bar{\lambda}$, which is the buckling load of the nonlinear, imperfect structure.

Slowly varying part of the deflection. For $\epsilon \rightarrow 0$ the buckling load $\lambda$ for the imperfect structure is expected to approach the buckling load $\lambda_{0}$ of the perfect structure. The buckling load $\lambda_{0}$ is the lowest eigenvalue of the homogeneous linear problem with solutions

$$
\lambda_{0}=\frac{1}{2}\left(\nu_{0}^{2}+\nu_{0}^{-2}\right), \quad \nu_{0}=n \pi / l, \quad w=\sin \nu_{0} x
$$

where $n$ is the integer that minimizes $\lambda_{0}$. For simplicity the length $l$ is taken to be equal to $n \pi$, which corresponds to $\lambda_{0}=1$ and $\nu_{0}=1$.

Let $\lambda=1-\delta^{2} / 2$, and since the buckling load $\bar{\lambda}$ will approach $\lambda_{0}=1$ for $\epsilon \rightarrow 0$, assume $\delta \ll 1$. Then rescaling the displacement $w=\delta \bar{w}$, and using $\lambda=1-\delta^{2} / 2$ leads to

$$
\begin{gathered}
\bar{w}^{\prime \prime \prime \prime}+2 \bar{w}^{\prime \prime}+\bar{w}=\delta^{2} \bar{w}^{\prime \prime}+\delta^{2} \bar{w}^{3}-2 \epsilon \lambda \delta^{-1} w_{0}^{\prime \prime}(x), \\
\bar{w}=\bar{w}^{\prime \prime}=0, \quad \text { for } \quad x= \pm n \pi .
\end{gathered}
$$

If one assumes $\epsilon \delta^{-1} \ll 1$ in the neighborhood of the buckling load, then Eq. (2) suggests that the solution $\bar{w}$ will be sinusoidal on a length scale 1 , with corrections that vary on a length scale much larger than 1. A set of Bogoliubov-type transformations [6] will be used to obtain equations for the slowly varying part of $\bar{w}$. I et

$$
\bar{w}=u_{1}(x) \cos x+u_{2}(x) \sin x
$$

where $u_{1}$ and $u_{2}$ are new unknown variables. Impose on $u_{1}$ and $u_{2}$ the condition

$$
u_{1}^{\prime} \cos x+u_{2}^{\prime} \sin x=0
$$

and introduce the variables $u_{3}$ and $u_{4}$

$$
u_{1}{ }^{\prime} \sin x-u_{2}{ }^{\prime} \cos x=\delta\left(u_{3} \sin x-u_{4} \cos x\right)
$$

which satisfy

$$
u_{3}{ }^{\prime} \sin x-u_{4}{ }^{\prime} \cos x=0 .
$$

Then the expressions for $\bar{w}^{\prime}, \bar{w}^{\prime \prime}$ and $\bar{w}^{\prime \prime \prime}$ are devoid of derivatives of $u_{i}$. Substitution of (3) into (2) produces a fourth equation in $u_{i}{ }^{\prime}$, in addition to Eqs. (4), (5), and (6). Solving these four equations for $u_{i}{ }^{\prime}$ leads to a system of first-order equations of the form

$$
\left.\begin{array}{r}
u_{i}{ }^{\prime}=\epsilon \lambda \delta^{-2} F_{i}{ }^{(0)}\left[w_{0}{ }^{\prime \prime}(x), \sin x, \cos x\right]+\delta F_{i}{ }^{(1)}[\mathbf{u}, \sin x, \cos x]+O\left(\delta^{2}\right) \\
i=1, \cdots, 4, \quad u_{1}=u_{4}=0 \text { at } x= \pm n \pi .
\end{array}\right\}
$$

This system of equations indicates that $u_{\imath}{ }^{\prime}$ is proportional to a small parameter, and that $u_{i}$ will be a slowly varying function of $x$, if $\epsilon \delta^{-2} \ll 1$. 
If the imperfection $w_{0}^{\prime \prime}(x)$ were deterministic and periodic, one could proceed now and use averaging methods $[6,7]$ to arrive at approximate equations for $u_{i}$. For example, with $w_{0}{ }^{\prime \prime}(x)=\sin x$, a constant first approximation of $u_{i}$ follows directly by averaging the right-hand sides of (7) over $x$. The buckling load $\bar{\lambda}$ associated with this first approximation is given by

$$
(1-\bar{\lambda})^{3 / 2}=2^{-5 / 2} 9 \bar{\lambda} \epsilon
$$

which agrees with the asymptotic results found in [2] by different methods. From (8) it also follows that $\bar{\lambda} \epsilon=0\left(\delta^{3}\right)$, which verifies the initial assumption $\epsilon \lambda \delta^{-2} \ll 1$.

Application of Khas'minskii's limit theorem. In [4] and [5] Khas'minskii extended the method of averaging to a class of random initial value problems. In [8] explicit relations are formulated between the probability density functions of boundary-value problems and corresponding initial-value problems. Using these relations, assuming existence of probability density functions for the displacement, one can show that Khas'minskii's limit theorem is also applicable to the boundary value problem (7), if the right-hand sides of (7) satisfy the appropriate conditions listed in [5].

Guided by the results obtained in $[1,2,3]$, assume tentatively that in $(7) \epsilon \lambda=0\left(\delta^{5 / 2}\right)$ for $\lambda=\bar{\lambda}$ and $\epsilon \rightarrow 0$, and write

$$
\epsilon \lambda=p \delta^{5 / 2} ; \quad p=0(1) \text { for } \lambda=\bar{\lambda} .
$$

Then the slowly varying part of the displacement $u_{i}$ satisfies the system of stochastic differential equations (7), which is now of the form

$$
\begin{gathered}
d u_{i} / d x=\delta^{1 / 2} F_{i}(\mathbf{u}, x), \\
F_{i}(\mathbf{u}, x)=F_{i}{ }^{(0)}\left[w_{0}{ }^{\prime \prime}(x), \sin x, \cos x\right]+\delta^{1 / 2} F_{i}{ }^{(1)}[\mathbf{u}, \sin x, \cos z]+0\left(\delta^{3 / 2}\right) .
\end{gathered}
$$

Let $w_{0}{ }^{\prime \prime}(x)$ be a bounded stationary random function with zero mean and correlation $\left\langle w_{0}(x) w_{0}(y)\right\rangle=R(x-y)$ and power spectrum

$$
S(\omega)=\frac{1}{2 \pi} \int_{-\infty}^{\infty} R(x) \exp (-i \omega x) d x .
$$

In addition, let $w_{0}{ }^{\prime \prime}(x)$ satisfy the conditions specified in [5], which imply that the shape of the imperfection of the column is statistically independent over sufficiently large distances. Then, $u_{i}$ converges for $\delta \rightarrow 0$ to the slowly varying process $u_{i}{ }^{(0)}(\xi)$ with $\xi=\delta x$, defined by

$$
\begin{aligned}
d u_{i}{ }^{(0)} / d \xi= & \Phi_{i}\left(u^{(0)}\right)+\sigma_{i m} g_{m}(\xi), \quad \xi=\delta x, \quad m=1, \cdots, 4 \\
& u_{1}{ }^{(0)}=u_{4}{ }^{(0)}=0, \quad \text { for } \quad \xi= \pm \delta n \pi
\end{aligned}
$$

where $g_{m}(\xi)$ are independent Gaussian white noise functions with $\left\langle g_{m}\right\rangle=0$, and $\left\langle g_{m}(\xi) g_{m}(\eta)\right\rangle=\delta_{m n} \delta(\xi-\eta)$. The quantities $\Phi_{i}\left[\mathfrak{u}^{(0)}\right]$ and $\sigma_{i m}$ are given by

$$
\begin{gathered}
\Phi_{i}(\mathfrak{u})=\lim _{L \rightarrow \infty} \frac{1}{L} \int_{0}^{L}\left\langle F_{i}{ }^{(1)}(\mathfrak{u}, x)\right\rangle d x, \\
\sigma_{i m} \sigma_{i m}=\alpha_{i i}, \\
\alpha_{i i}=\lim _{L \rightarrow \infty} \frac{1}{L} \int_{0}^{L} \int\left\langle F_{i}{ }^{(0)}(x) F_{i}{ }^{(0)}(y)\right\rangle d x d y .
\end{gathered}
$$


Performing the averaging operations of (11) and (12), one finds for (10)

$$
\begin{aligned}
& d u_{1}{ }^{(0)} / d \xi=\frac{1}{2} u_{3}{ }^{(0)}, \\
& d u_{2}{ }^{(0)} / d \xi=\frac{1}{2} u_{4}{ }^{(0)}, \\
& d u_{3}{ }^{(0)} / d \xi=\frac{1}{2} u_{1}{ }^{(0)}\left[1-\frac{3}{4}\left(u_{1}{ }^{(0) 2}+u_{2}{ }^{(0) 2}\right)\right]+2 p[\pi S(1)]^{1 / 2} g_{3}(\xi), \\
& d u_{4}{ }^{(0)} / d \xi=\frac{1}{2} u_{2}{ }^{(0)}\left[1-\frac{3}{4}\left(u_{1}{ }^{(0) 2}+u_{2}{ }^{(0) 2}\right)\right]+2 p[\pi S(1)]^{1 / 2} g_{4}(\xi), \\
& u_{1}{ }^{(0)}=u_{4}{ }^{(0)}=0 \text { for } \xi= \pm \delta n \pi .
\end{aligned}
$$

Note that in this asymptotic set of equations for $u_{i}$ the process $w_{0}{ }^{\prime \prime}(x)$, which is uncorrelated over long distances $x$, is replaced by a Gaussian process which is delta-correlated on a length scale $\xi=\delta x$. This result can best be understood if Khas'minskii's limit theorem is seen as a generalization of the classical central limit theorem to processes that are functionals of asymptotically independent processes defined via differential equations [9].

Also note that $u_{i}{ }^{(0)}$ depends on the imperfection shape $w_{0}{ }^{\prime \prime}(t)$ only through the power spectrum evaluated at unity. This corresponds to the buckling mode of the perfect structure which has a wave number unity.

The replacement of the random functions $w_{0}^{\prime \prime}(x)$ in (9) by Gaussian forcing functions in (13) opens the possibility of applying equivalent linearization or perturbation techniques used in $[1,2,3]$ to obtain approximate asymptotic results for the maximum load $\bar{\lambda}$.

Maximum load. A first approximation for the maximum load $\hat{\lambda}$ is obtained by using a perturbation scheme which is similar to the one used by Amazigo [3] for the case of Gaussian imperfection shapes. For fixed $\lambda$, the coefficient $p=\epsilon \lambda \delta^{-5 / 2}$ in (13) goes to zero for $\epsilon \rightarrow 0$. Then according to (13), also $u_{i}$ will approach zero for $\epsilon \rightarrow 0$ and $\lambda<\bar{\lambda}$.

Write $p(2 \pi S(1))^{1 / 2}=\epsilon q$, and restate the system of equations (13) for the approximate boundary-value problem in terms of two coupled integral equations for $u_{1}{ }^{\left({ }^{0}\right)}$ and $u_{2}{ }^{(0)}$ :

$$
\begin{aligned}
& u_{1}(\xi)=L^{-1}(\xi, \eta)\left\{2^{-1 / 2} \epsilon q g_{3}(\eta)-(3 / 16) u_{1}(\eta)\left[u_{1}{ }^{2}(\eta)+u_{2}{ }^{2}(\eta)\right]\right\}, \\
& u_{2}(\xi)=L^{-1}(\xi, \eta)\left\{2^{-1 / 2} \epsilon q g_{4}(\eta)-(3 / 16) u_{2}(\eta)\left[u_{1}{ }^{2}(\eta)+u_{2}{ }^{2}(\eta)\right]\right\} .
\end{aligned}
$$

In these equations the superscripts zero have been dropped. For convenience the length of the column will be chosen large enough that the influence of the boundary conditions on the maximum load can be neglected. Thus, the operator $L^{-1}$ is defined as

$$
L^{-1}(\xi, \eta)\{\}=\int_{-\infty}^{\infty} G(\xi,-\eta)\{\} d \eta
$$

where the Green's function $G(\xi)$ is the bounded solution of the linear problem

$$
d^{2} G / d \xi^{2}-\frac{1}{4} G=\delta(\xi) .
$$

Expand $u_{1}$ and $u_{2}$ in powers of $\epsilon q$ according to

$$
\begin{aligned}
& u_{1}=\sum_{n=1}(\epsilon q)^{2 n-1} a_{n}(\xi), \\
& u_{2}=\sum_{n=1}(\epsilon q)^{2 n-1} b_{n}(\xi) .
\end{aligned}
$$

Substituting these expansions into the integral equation (14) and equating powers 
of $\epsilon q$ gives

$$
\begin{array}{ll}
a_{1}=2^{-1 / 2} L^{-1}\left\{g_{3}\right\}, & b_{1}=2^{-1 / 2} L^{-1}\left\{g_{4}\right\}, \\
a_{2}=-(3 / 16) L^{-1}\left\{a_{1}{ }^{3}+a_{1} b_{1}{ }^{2}\right\}, & b_{2}=-(3 / 16) L^{-1}\left\{b_{1}{ }^{3}+b_{1} a_{1}{ }^{2}\right\}, \\
\text { etc. } & \text { etc. }
\end{array}
$$

According to (17) and the definition of the white noise functions $g_{m}(\xi), a_{1}(\xi)$ and $b_{1}(\xi)$ are two equal but independent Gaussian random functions with correlations

$$
\begin{aligned}
& \left\langle a_{1}(\xi) a_{1}(\eta)\right\rangle=\left\langle b_{1}(\xi) b_{1}(\eta)\right\rangle=\frac{1}{2} \int G\left(\xi-\eta^{\prime}\right) G\left(\eta-\eta^{\prime}\right) d \eta^{\prime}, \\
& \left\langle a_{1}(\xi) b_{1}(\eta)\right\rangle=0 .
\end{aligned}
$$

Furthermore, from (16) and (17) it follows by induction that $u_{1}$ and $u_{2}$ are orthogonal with correlations

$$
\left\langle u_{1}(\xi) u_{1}(\eta)\right\rangle=\left\langle u_{2}(\xi) u_{2}(\eta)\right\rangle, \quad\left\langle u_{1}(\xi) u_{2}(\eta)\right\rangle=0 .
$$

Let $\Delta^{2}$ be the mean square of the deflection $\bar{w}(x)$; then, according to (19),

$$
\Delta^{2} \equiv\left\langle\bar{w}^{2}\right\rangle=\left\langle\left(u_{1} \cos x+u_{2} \sin x\right)^{2}\right\rangle=\left\langle u_{1}{ }^{2}\right\rangle,
$$

and using the series expansions (16)

$$
\Delta^{2}=\left\langle a_{1}{ }^{2}\right\rangle(\epsilon q)^{2}+2\left\langle a_{1} a_{2}\right\rangle(\epsilon q)^{4}+\left(\left\langle a_{2}{ }^{2}\right\rangle+2\left\langle a_{1} a_{3}\right\rangle\right)(\epsilon q)^{6}+0\left(\epsilon^{8} q^{8}\right) .
$$

Since $q$ is a function of $\lambda$, Eq. (20) gives a relation between $\lambda, \epsilon$ and $\Delta^{2}$. The expansion is expected to converge asymptotically for $\epsilon \rightarrow 0$ and $\lambda<\bar{\lambda}$. However, $\Delta^{2}$ is a multivalued function of $\lambda$ and this representation for $\Delta^{2}$ breaks down for $\lambda>\bar{\lambda}$. In Fig. 1 the expected behavior of $(\epsilon q)^{2}$ is sketched as a function of $\Delta^{2}$.

As in [3], the singularity of $\Delta^{2}$ at $\lambda=\bar{\lambda}$ is avoided by considering the inverse problem which gives $\lambda$ or $(\epsilon q)^{2}$ as analytic functions of $\Delta^{2}$ in the neighborhood of $\lambda=\bar{\lambda}$ (see Fig. 1).

$$
(\epsilon q)^{2}=\beta_{1} \Delta^{2}+\beta_{2} \Delta^{4}+\beta_{3} \Delta^{6}+0\left(\Delta^{8}\right) .
$$

Substituting (21) into (20) and equating powers of $\Delta^{2}$ leads to expressions for the coefficients $\beta_{i}$. In particular, we have

$$
\beta_{1}=\left\langle a_{1}^{2}\right\rangle^{-1}, \quad \beta_{2}=-2\left\langle a_{1} a_{2}\right\rangle\left\langle a_{1}{ }^{2}\right\rangle^{-3} .
$$

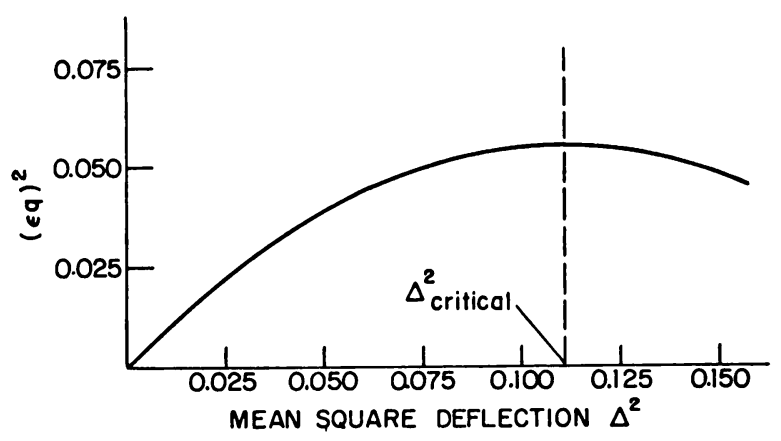

Fig. 1. $(\epsilon q)^{2}$ versus mean square displacement. 
Use of Eq. (21) and the definitions $q=(2 \pi S)^{1 / 2} \delta^{-5 / 2} \lambda$ and $\delta=[2(1-\lambda)]^{1 / 2}$ gives $\lambda$ as a function of $\Delta^{2}, \epsilon$ and $S(1)$ :

$$
(1-\lambda)^{5 / 4}=\left[f\left(\Delta^{2}\right)\right]^{-1 / 2} 2^{-5 / 4}[2 \pi S(1)]^{1 / 2} \epsilon \lambda
$$

where $f\left(\Delta^{2}\right)$, which is independent of $\epsilon, \lambda$, and $S(1)$, is given by the right-hand side of (21).

The maximum load $\bar{\lambda}$ then follows by maximizing $f$ with respect to $\Delta^{2}$. For a first approximation of $\bar{\lambda}$ retain only two terms in the expansion for $f\left(\Delta^{2}\right)$. Setting $d f / d \Delta^{2}=0$ and using (22) leads to

$$
\bar{f} \sim\left\langle a_{1}{ }^{2}\right\rangle / 8\left\langle a_{1} a_{2}\right\rangle
$$

According to (17),

$$
\left\langle a_{1} a_{2}\right\rangle=-(3 / 16) \int_{-\infty}^{\infty} G(\xi-\eta)\left\{\left\langle a_{1}{ }^{3}(\eta) a_{1}(\xi)\right\rangle+\left\langle a_{1}(\eta) a_{1}(\xi) b_{1}{ }^{2}(\eta)\right\rangle\right\} d \eta
$$

Since $a_{1}$ and $b_{1}$ are two equal but independent Gaussian random variables, it follows that

$$
\left\langle a_{1}{ }^{3}(\eta) a_{1}(\xi)\right\rangle=3\left\langle a_{1}{ }^{2}\right\rangle\left\langle a_{1}(\eta) a_{1}(\xi)\right\rangle, \quad\left\langle a_{1}(\eta) a_{1}(\xi) b_{1}{ }^{2}(\eta)\right\rangle=\left\langle a_{1}{ }^{2}\right\rangle\left\langle a_{1}(\eta) a_{1}(\xi)\right\rangle .
$$

The right-hand side of (24) can now easily be calculated, using (18) and the definition of the Green's function (15). Substitution of the resulting value for the critical $\bar{f}$ into (23) gives for the maximum load $\bar{\lambda}$

$$
(1-\bar{\lambda})^{5 / 4} \sim 3(2)^{-3 / 4}[2 \pi S(1)]^{1 / 2} \epsilon \bar{\lambda} .
$$

This approximate asymptotic result for the buckling load $\bar{\lambda}$ as a function of the amplitude $\epsilon$ and the spectral density $S(1)$ of the imperfection, is the same as found in [3] for Gaussian imperfections. It also varifies the initial assumption $\epsilon \lambda=0\left(\delta^{5 / 2}\right)$ at $\lambda=\bar{\lambda}$.

Discussion of the results. Eq. (13) for the slowly-varying part $u_{t}$ of the deflection of the column is asymptotically exact. One may expect also that Eq. (23), which relates the load parameter $\lambda$ to the imperfection parameter $\epsilon$ and $S(1)$, is asymptotically exact for $\epsilon \rightarrow 0$ and given $f\left(\Delta^{2}\right)$. The result (25) for the buckling load $\bar{\lambda}$, however, is approximate in the sense that the accuracy of the two-term approximation used to calculate the critical coefficient $\bar{\lambda}$ does not improve for $\epsilon \rightarrow 0$. Because of the asymptotic nature of the analysis and the rapid escalation of the algebra involved, no attempt has been made to improve on the first approximation (24) through retention of more terms in the expansion for $f\left(\Delta^{2}\right)$ in (22). As reported in the analogous analysis [3], a three-term approximation for the case of Gaussian imperfection shapes did not significantly change the coefficient in (24).

Comparing (23) and (25) with (8) leads to the conclusion that for a large range of random imperfection shapes the deterioration of the buckling strength, as measured by the dependence on $\epsilon$, is less severe for random than for sinusoidal imperfections.

\section{RefERENCES}

[1] W. B. Fraser and B. Budiansky, The buckling of a column with random initial deflections, J. Appl. Mech. 36, 232-240 (1969)

[2] J. C. Amazigo, B. Budiansky and G. F. Carrier, Asymptotic analysis of the buckling of imperfect columns on nonlinear elastic foundations, Int. J. Solids Struct. 6, 1341-1356 (1971)

[3] J. C. Amazigo, Buckling of stochastically imperfect columns on nonlinear elastic foundations, Quart. Appl. Math. 29, 403-410 (1971) 
[4] R. Z. Khas'minskii, On stochastic processes defined by differential equations with a small parameter, Theory Prob. Applic. 211-228 (1966)

[5] R. Z. Khas'minskii, $\boldsymbol{A}$ limit theorem for the solution of differential equations with random right-hand sides, Theory Prob. Applic., 390-406 (1966)

[6] N. Krylov and N. Bogoliubov, Introduction to non-linear mechanics, Princeton University Press, Princeton, New Jersey, 1947

[7] N. N. Bogoliubov and Y. A. Mitropolsky, Asymptotic methods in the theory of non-linear oscillations, Hindustan Publishing Co., Delhi, India, 1961

[8] B. P. Videc, Nonlinear stochastic boundary value problems for ordinary differential equations, Ph.D. Thesis, Harvard University, Cambridge, Mass., 1974

[9] G. C. Papanicolaou, Stochastic equations and their applications, Amer. Math. Monthly 80, 542 (1973) 